\title{
New Methodology to Evaluate Sunscreens Under Outdoor Conditions: A Double-Blind, Randomized Intra-Individual Clinical Study of a Water-Based Broad-Spectrum SPF50+ Versus SPF15 (P3) and SPF50+
}

\author{
Corinne Granger (D) - Jean Krutmann · Javier Bustos · Yolanda Sola • \\ Muzzammil Hosenally · Carles Trullàs · Philippe Andres • \\ Thierry Passeron
}

Received: July 1, 2019 / Published online: July 27, 2019

(C) The Author(s) 2019

\begin{abstract}
Introduction: This study explored a new method to test sunscreens in outdoor conditions (very high to extreme ultraviolet [UV] radiation) approximating real-life solar exposure while maintaining scientific standards and acceptable conditions, and assessed the efficacy of a water-based sun-protection factor (SPF) 50+ versus a reference SPF15 and two comparator SPF50+ products.
\end{abstract}

Enhanced Digital Features To view enhanced digital features for this article go to https://doi.org/10.6084/ m9.figshare.8952746.

Electronic supplementary material The online version of this article (https://doi.org/10.1007/s13555019-00315-4) contains supplementary material, which is available to authorized users.

C. Granger $(\varangle) \cdot J$. Bustos · C. Trullàs Innovation and Development, Isdin, Barcelona, Spain

e-mail: corinne.granger@isdin.com

J. Krutmann

IUF-Leibniz Research Institute for Environmental

Medicine, Düsseldorf, Germany

Y. Sola

Meteorology Group, Department of Applied Physics, University of Barcelona, Martí I Franquès 1, 08028

Barcelona, Spain

M. Hosenally

Department of Economics and Statistics, University of Mauritius, Réduit, Mauritius
Methods: Thirty-five subjects underwent testing in summertime Mauritius. In each subject, five test areas were marked on the back. One area was left unprotected, and four sunscreens were applied to the others: investigational product (IP), SPF15 (ISO 24444:2010 reference standard P3), and two marketed SPF50+ controls. Subjects spent $1-2 \mathrm{~h}$ (depending on skin type) in the sun. After $24 \mathrm{~h}$, erythema was assessed by clinical scoring (0-5) and colorimetry $\left(a^{*}, L^{*}\right.$, and ITA). Secondary endpoints were correlation between clinical and colorimetry assessment, product tolerability, and total UV radiation received.

Results: All subjects were exposed to a very high UV index $(\geq 8)$ and $30 / 35$ were exposed to an extreme UV index $(\geq 11)$. The IP showed statistically significant differences in clinical erythema scoring compared with unprotected

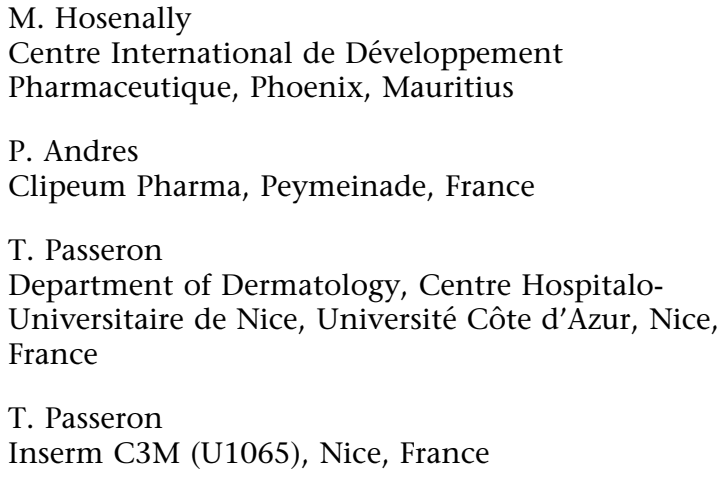


skin and SPF15, but not with SPF50+ controls. On colorimetry, differences in $a^{*}$ (redness) and $L^{*}$ (lightness) reached statistical significance for the IP vs SPF15 but not vs SPF50+ controls. Clinical and instrumental erythema assessment correlated strongly (Spearman's rho 0.663). No tolerability issues were reported.

Conclusion: This exploratory study confirmed the ability of this outdoor model to discriminate sunscreens with different SPF using clinical evaluation as an objective measure. The waterbased sunscreen maintained its efficacy in outdoor conditions of very high to extreme UV radiation: it was superior to SPF15 and comparable to SPF50+ controls in preventing erythema. The method used represents an option for sunscreen efficacy comparison outside of the laboratory.

Funding: Isdin.

Keywords: Outdoor; SPF; Sun protection; Sunburn

\section{INTRODUCTION}

Solar ultraviolet (UV) radiation is one of the most important environmental factors affecting skin physiology, with short- and long-term consequences including actinic erythema (sunburn), inflammatory responses, photoaging, photo-immunosuppression, and skin cancers $[1,2]$. Recommended protective measures include seeking shade, wearing protective clothing, hats, and sunglasses, and using sunscreens. In reality, sunscreens often form the mainstay of sun protection strategies, and sun protection is frequently suboptimal [3].

Standardized methods have been established for in vivo laboratory testing of sun protection factor (SPF) $[4,5]$, which corresponds to the level of protection against UV-induced erythema. SPF is the main information presented when labelling sunscreens, but it has some drawbacks. It is primarily a measure of UVB protection (and to a lesser extent, UVA2), yet it is now demonstrated that UVA1, visible, and infrared (IR) radiation also have potential negative effects on the skin [2]. The methods defined in SPF testing guidelines do not fully reproduce conditions of real solar exposure, mainly because the solar simulators used do not replicate the full sunlight spectrum and deliver high UV intensities over a short time; determination of protection against UVA radiation requires additional testing [6].

Besides the radiation conditions, SPF testing furthermore does not take into account the effect of two factors known to affect topical formulations, namely external temperature and humidity. Outdoor studies to evaluate the photoprotective effect of sunscreens under real conditions of exposure are seldom reported. Such studies are difficult to standardize and expensive.

We developed a water-based sunscreen (WBS) designed to avoid leaving unpleasant greasy or white residues on the skin. The product was determined as SPF50+ on in vivo laboratory testing (ISO 24444: 2010) [4]. Since this novel WBS formulation had fewer supporting data on its efficacy performance than older, traditional, oil-based sunscreens, we wanted to have further information on its photoprotective properties beside its high laboratory-assessed SPF. We previously conducted an initial outdoor study on the efficacy of the WBS, which used a split-face design to assess solar-induced erythema in a Mediterranean setting [7]. Following on from this, we wanted to assess its efficacy in a setting with more extreme solar radiation, incorporating the full solar spectrum, and in which external temperatures and humidity could potentially exert an effect, to assess if the WBS maintained its protective capacity. We report here on the methodology used and the efficacy results.

\section{METHODS}

\section{Tested Products}

The investigational product was a water-based SPF50+ sunscreen (WBS). This was compared against three control sunscreens: the ISO2444:2010 reference standard sunscreen P3 and two different commercially available SPF50+ sunscreens. The UVB and UVA filters 
included in the different products are described in the supplementary material (Appendix 1).

\section{Subjects}

Thirty-five healthy male and female volunteers were recruited. Participants were required to be aged 18-45 years old, have skin phototype II to IV [based on individual typology angle (ITA) measurements] with no pigmentation or photoaging disorders, excess hair or nevi, history of skin cancer, or intensive sun exposure within 3 months prior. Concomitant medications judged by the investigator as having the potential to interfere with the study, namely anti-inflammatories and photosensitizing drugs (thiazides, quinolones, tetracyclines, fluoroquinolones, topical and oral vitamin A derivatives, psoralens, and amino levulinic acid derivatives) were not allowed.

\section{Study Design}

This clinical study was conducted as a singlecenter, double-blind, randomized intra-individual study, in December (summer) 2018 in Mauritius. The study was approved (28 November 2018) by an independent ethics committee (Independent Ethics Committee, King George V Corner, Floreal). The study was conducted in line with the principles of the Declaration of Helsinki and its subsequent updates, the International Conference on Harmonization Good Clinical Practices, and local laws. All subjects provided written informed consent to participate.

\section{Product Application and Solar Exposure}

Five $12 \mathrm{~cm}^{2}$ rectangular areas were marked on volunteers' backs with medical tape. Thirty minutes before exposure, a technician applied four sunscreens (WBS, SPF15, and two SPF50+ controls) to their respective test areas, according to a randomization list and at a dose of $2 \mathrm{mg} /$ $\mathrm{cm}^{2}$. The fifth area was left as an untreated control. The identity of the products applied to each area was blinded to the technician and the investigator who scored erythema. The unprotected area was unblinded to the investigator for safety reasons: any cases of grade 2 erythema before the end of the study period were to be covered. Non-investigational areas were protected with hats, sunglasses, and UVprotective tee shirts (Fig. 1).

Thirty minutes after sunscreen application, subjects lay down outdoors, face-down with the test areas on their back exposed to the sun (a poolside location in Tamarin city). Subjects with phototype II skin spent $1 \mathrm{~h}$ in this position and those with phototype III or IV spent $2 \mathrm{~h}$. This difference in exposure times was to minimize the risk of severe sunburn: the original study design saw subjects exposed for $2 \mathrm{~h}$, but given that some subjects in the first group of volunteers developed erythema on all test areas and thus were not considered sufficiently protected even with SPF50+ products, a safety committee organized by the contract research organization implemented the above limit of $1 \mathrm{~h}$ on exposure time for those with skin phototype II.

After the exposure period, no further sunlight exposure to the back was permitted until the study assessment at $24 \mathrm{~h}$ had been completed. Subjects were allowed to shower but not exfoliate or apply cream to the study area.
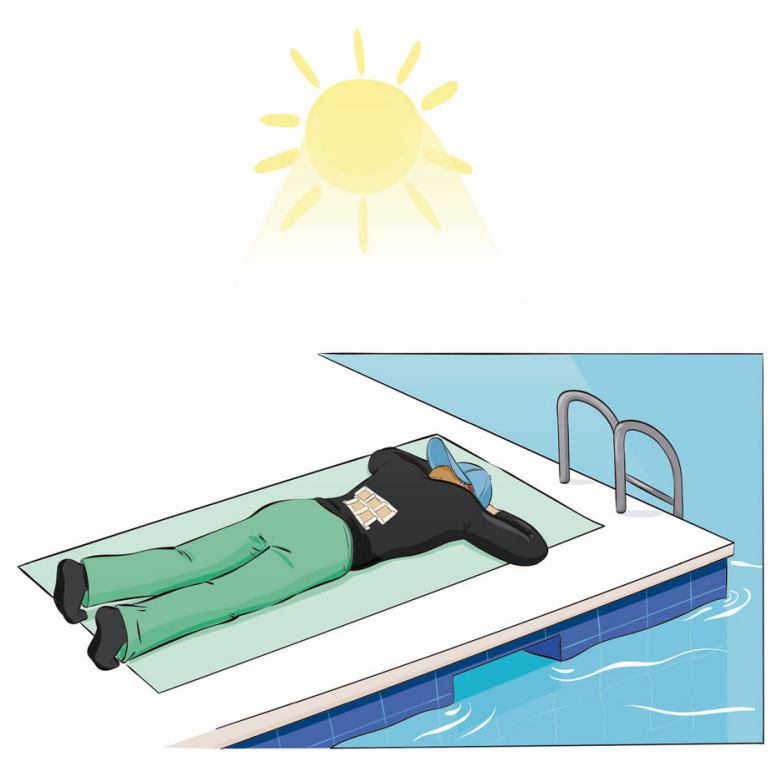

Fig. 1 Illustration of a study subject lying face-down with the test areas marked on the back and non-study areas protected with clothing 


\section{Evaluation of Erythema: Clinical Scoring}

The primary endpoint was clinical erythema score at $24 \mathrm{~h}$ after UV exposure, according to a 6-point scale: $0=$ no erythema; $1=$ equivocal reaction, slight, barely perceptible erythema (not clearly defined); 2 = clearly visible erythema with well-defined borders; $3=$ moderate erythema; $4=$ severe erythema; 5 = very severe erythema with blistering (Appendix 2 in the supplementary material) [8]. Erythema was assessed at baseline and at $24 \mathrm{~h}$ by a trained technician under the supervision of a certified dermatologist. Subjects stayed in a temperatureand hygrometry-controlled room for at least 15 min before assessment.

\section{Evaluation of Skin Colorimetry}

Secondary endpoints of the colorimetry parameters $a^{*}$ (indicative of redness, higher values indicating redder skin), $L^{*}$ (indicative of pigmentation, lower values indicating greater pigmentation), and ITA (individual typology angle, calculated according to the following formula: $\left.\left\{\arctan \left[\left(L^{*}-50\right) / b^{*}\right]\right\} \times 180 / \pi\right)$ were analyzed at baseline and at $24 \mathrm{~h}$. The Chroma Meter CR400 (Konica Minolta, Inc., Tokyo, Japan) was used in accordance with the manufacturer's instructions, placed gently on the skin, taking measurements rapidly and repositioning the device by a few millimeters between flashes, then calculating the mean of three readings. Individual $b^{*}$ values (yellow-blue balance on colorimetry reading) were used only for ITA calculation.

For colorimetry values, results were also compared against an unexposed area of skin on the back, which was marked to ensure the same area at each reading.

\section{Tolerance and Safety}

Skin tolerance after a single application of the products was assessed by a technician under the supervision of a certified dermatologist with experience in skin tolerance testing at 1,2, and $24 \mathrm{~h}$.
Clinical erythema was also scored at 1 and $2 \mathrm{~h}$ for safety purposes, and any areas with a score $>2$ were to be covered.

\section{Erythemal Solar Irradiance}

Ultraviolet radiation data was analyzed by the Meteorology Group of the Department of Applied Physics, University of Barcelona. Estimated solar spectral irradiance was weighted with the erythemal action spectrum. The solar spectral irradiance for cloud-free conditions was estimated through a radiative transfer model that takes into account the day of the year, time of day, latitude, longitude, altitude, and thickness of the ozone layer. The dose of UV erythemal radiation received in millijoules per square centimeter was calculated by pairing solar erythemal irradiance and exposure time.

\section{Statistical Analysis}

Analysis was performed on an intention-to-treat basis, meaning any subjects who received product application and UV exposure. Quantitative variables were reported as measures of central tendency (mean, median, minimum, and maximum) and dispersion (standard deviation [SD]). Qualitative variables were reported as frequencies and percentages.

For the parameters derived from clinical scoring and colorimetry, univariate ANOVA was performed with "product" as the fixed factor and "subject" as the random factor, followed by Tukey's procedure for pairwise comparisons. Analyses were conducted on absolute values and on percentage difference with respect to the unprotected area at $24 \mathrm{~h}$. Analysis was conducted on rank transformed data whenever the Shapiro-Wilk test (at 1\%) indicated that the assumption of normality was not met. $P$ values less than 0.05 were considered significant.

As a measure of the accuracy of the methodology, we also assessed the correlation between clinical erythema scores and colorimetry ( $a^{*}$ parameter $)$ using Spearman's ranked correlation coefficient. 


\section{RESULTS}

\section{Subject Characteristics}

Sixteen men and 19 women participated; all were Caucasian. Mean (SD) age was 25 (7) years, minimum 18-maximum 44 years. Based on ITA values, the distribution by phototype was 1 patient with type I (an inclusion error), 12 patients with type II, 13 with type III, and 9 with type IV (type I, ITA > 55; type II, 42-55; type III, 29-41; type IV, 11-28).

\section{Erythemal Solar Irradiance}

The mean hourly UV radiation was $103.47 \mathrm{~mJ} /$ $\mathrm{cm}^{2}$. The minimum total UV radiation was $89.20 \mathrm{~mJ} / \mathrm{cm}^{2}$ ( $1 \mathrm{~h}$ of exposure for type II subjects) and the maximum was $256.35 \mathrm{~mJ} / \mathrm{cm}^{2}$ ( $2 \mathrm{~h}$ of exposure). All participants were exposed to a very high UV index ( $\geq 8)$, and 30/35 participants were exposed to an extreme UV index $(\geq 11)$.

\section{Erythema: Clinical Scores}

Photographs of the five test areas from one subject are shown in Fig. 2. Mean erythema scores at $24 \mathrm{~h}$ are shown in Fig. 3, with full data reported in Table 1 . There was a statistically significant difference between the WBS and the unprotected area (mean difference in score, $-2.3 ; P<0.001$ ), and versus the SPF15 (mean difference, $-0.60 ; P<0.001)$. There was no statistically significant difference versus either of the two SPF50+ controls.

Similarly, when we compared products according to the mean percentage difference versus unprotected skin, there was a statistically significant difference between the WBS and the SPF15 control, but not between the WBS and either of the SPF50+ controls (Table 1).

Frequency distribution of erythema scoring is presented in Table 2 . The majority of subjects $(\geq 24)$ scored 0 for the areas protected with the WBS or SPF50+ controls; for the SPF15 areas, the scores were more spread between 0 and 2;

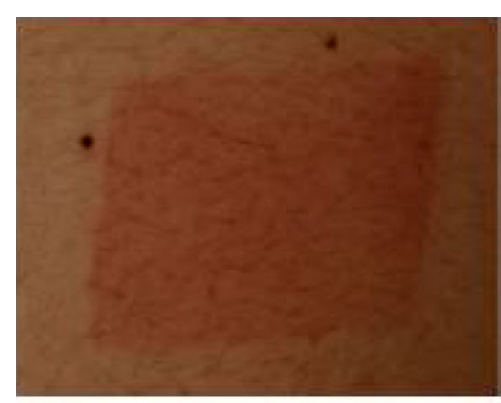

UP

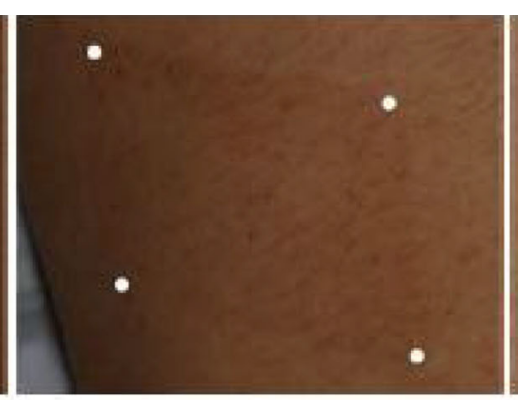

WBS

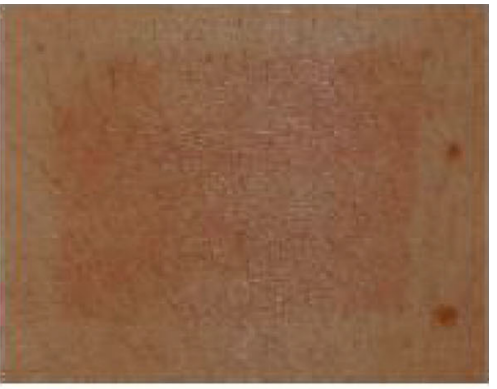

SPF15 (P3)

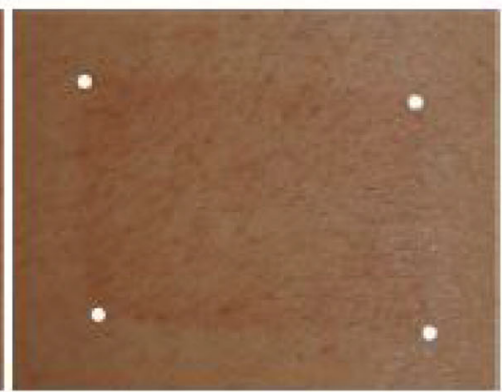

SPF50+ (2)

Fig. 2 Five skin test areas in one subject. The white dots mark the edges of the test areas in those areas that had milder erythema. SPF sun protection factor, UP unprotected control, WBS water-based sunscreen 


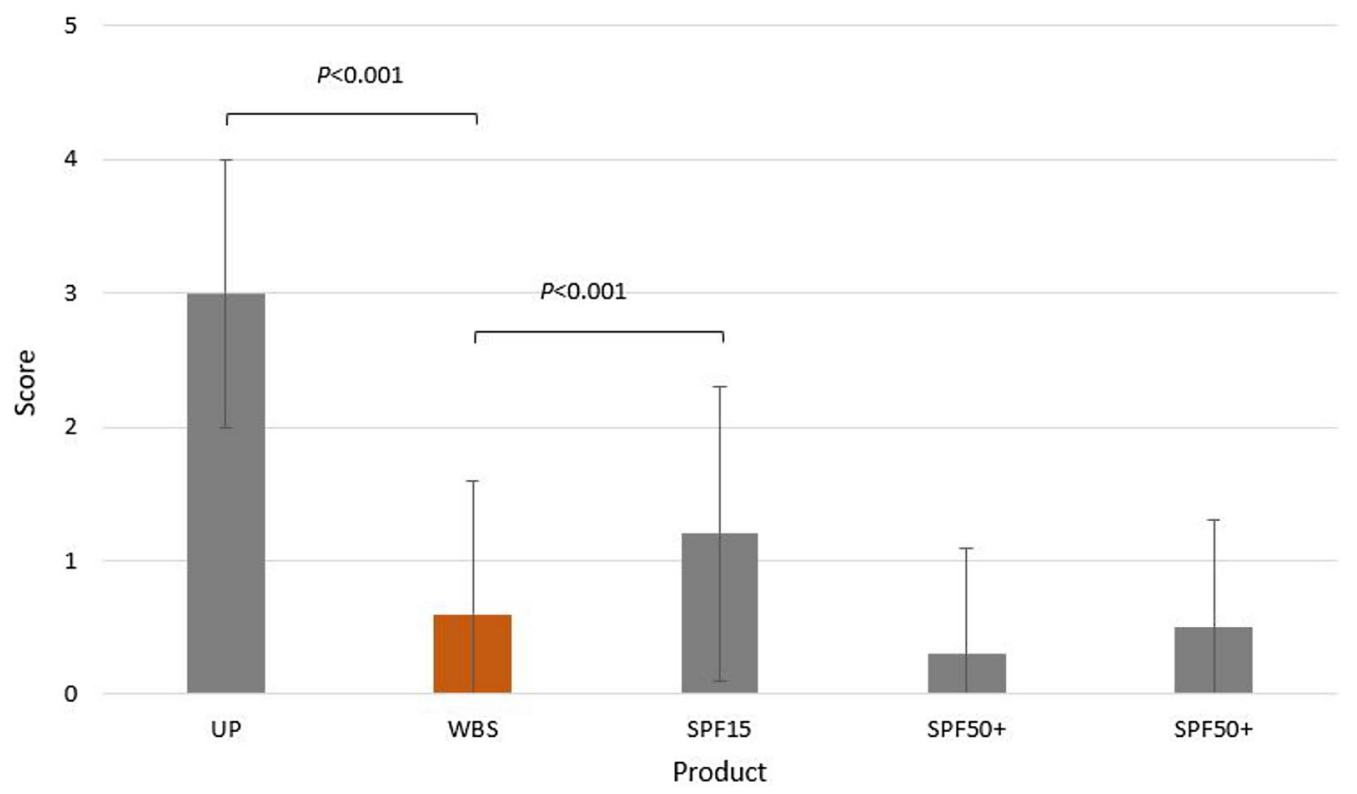

Fig. 3 Mean (SD) clinical erythema score at $24 \mathrm{~h}$, for each product. $S P F$ sun protection factor, $W B S$ water-based sunscreen

for the unprotected area, many subjects (27) had scores $\geq 3$, confirming the high UV index.

\section{Colorimetry}

At $24 \mathrm{~h}$, there was a statistically significant difference in mean absolute $a^{*}$ values (redness) between the WBS $(11.38 \pm 3.25)$ and the unprotected area $(17.59 \pm 3.37) \quad(P<0.001)$. The area treated with SPF15 gave higher $a^{*}$ values $(12.42 \pm 3.60)$ than the area treated with the WBS, indicative of redder skin, but the difference was not statistically significant.

Assessing the mean percentage differences from unprotected skin, for $a^{*}$, the WBS $(-34.51 \% \pm 17.46 \%)$ was significantly different to the SPF15 $(-29.10 \% \pm 16.89 \%)$ $(P=0.011)$, but not to either of the SPF50+ controls $(-36.95 \% \pm 18.76 \%$ and $-36.50 \% \pm$ $20.58 \%$, respectively) (Fig. 4). There was also a statistically significant difference in $L^{*}$ (pigmentation) between the WBS $(+8.76 \% \pm$ $4.98 \%)$ and the SPF15 $(+6.95 \% \pm 4.63 \%)$ $(P=0.030)$ but again, not with either of the SPF50+ comparators $(+9.01 \% \pm 5.59 \%$ and $+9.08 \% \pm 5.10 \%$, respectively).

For ITA, there was a statistically significant difference between the WBS and the unprotected and unexposed areas, but not between the WBS and any of the control products.

\section{Correlation Between $\boldsymbol{a}^{*}$ and Clinical Erythema Score}

On the basis of 175 measurements, there was a positive correlation between $a^{*}$ and clinical erythema score: the correlation coefficient (Spearman's rho) was 0.663.

\section{Safety Parameters}

All products (investigational and control) were well tolerated with no local tolerance issues.

\section{DISCUSSION}

This study was designed to explore a new methodology to evaluate the photoprotective effect of sunscreens in outdoor conditions. It compared a water-based broad-spectrum SPF50+ against three existing products: a reference standard SPF15 (P3) and two comparator SPF50+ products. The comparisons were performed under conditions of very high to 


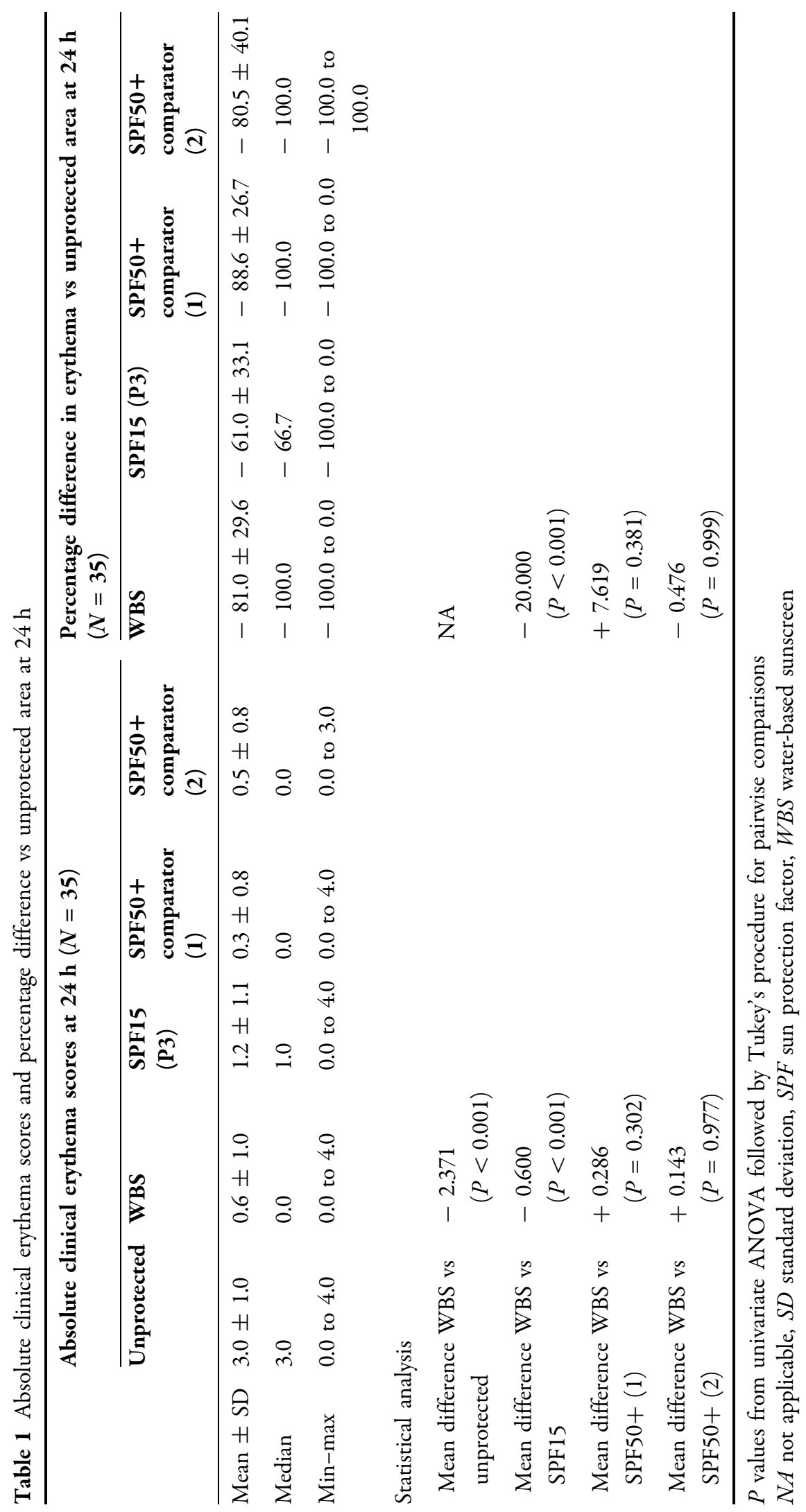


Table 2 Frequency distribution of erythema scoring at $24 \mathrm{~h}$

\begin{tabular}{llllll}
\hline \multirow{2}{*}{ Erythema score } & \multicolumn{5}{l}{ Number of subjects } \\
& Unprotected & WBS & SPF15 (P3) & SPF50+ comparator (1) & SPF50+ comparator (2) \\
\hline 0 & 1 & 23 & 12 & 29 & 24 \\
1 & 3 & 5 & 10 & 3 & 7 \\
2 & 4 & 6 & 8 & 2 & 3 \\
3 & 15 & 0 & 4 & 0 & 1 \\
4 & 12 & 1 & 1 & 1 & 0 \\
5 & 0 & 0 & 0 & 0 & 0 \\
\hline
\end{tabular}

$S P F$ sun protection factor, $W B S$ water-based sunscreen

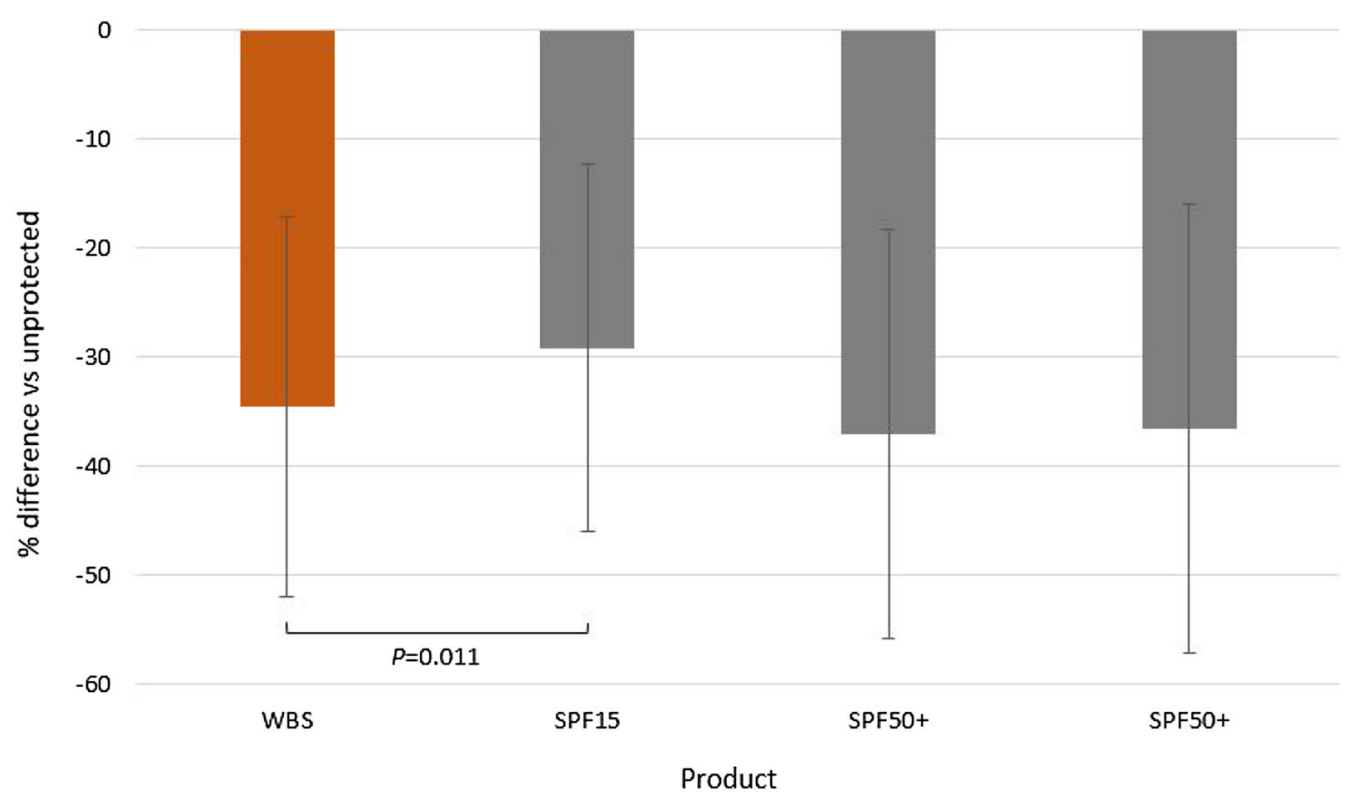

Fig. 4 Mean (SD) percentage difference in $a^{*}$ vs unprotected area at $24 \mathrm{~h} . S P F$ sun protection factor, $W B S$ water-based sunscreen

extreme solar radiation, with all 35 subjects exposed to a UV index $\geq 8$ and 30/35 exposed to a UV index $\geq 11$. We found that the WBS was consistently superior to the SPF15 reference and comparable to the two SPF50+ controls, as shown on clinical erythema score and colorimetry parameters $a^{*}$ and $L^{*}$. This was in line with the previously reported laboratory findings of a very high level of sun protection (SPF50+). While this finding was anticipated on the basis of previous laboratory testing and previous clinical outdoor split-face studies [7], we confirmed that despite the water-based formulation of the product tested, its efficacy performance was similar to that of older, traditional, oil-based sunscreens. Thus, it is reassuring to observe that its efficacy is maintained under these conditions.

As would be expected under such conditions of very high to extreme UV radiation, all except one subject had erythema at $24 \mathrm{~h}$ on the unprotected area $(31 / 35$ subjects scored $\geq 2)$. No grade 5 erythema was reported in any of the test areas, including the unprotected area 
(which was often covered at $1 \mathrm{~h}$ ). This point is reassuring from an ethical and medical perspective, as it confirms that the study design did not expose subjects to severe sunburn. Further improvement of the model would include a reduction of the unprotected area in order to limit sunburn to the minimal needed for clinical assessment and colorimetry measurements.

The low erythema scores for the areas treated with the WBS demonstrated its high photoprotection, comparable to the two other SPF50+ products. In contrast, the scores for the SPF15 had a broader distribution and indicate that this level of photoprotection was insufficient under such extreme conditions. Interestingly, the subject who had grade 4 erythema with the WBS also scored 3-4 for the two SPF50+ control products. This subject, with phototype II skin, was exposed for $2 \mathrm{~h}$ to extreme solar radiation and was not protected by any test product. This population represents an ongoing challenge in skin care, and requires careful user education regarding other photoprotection measures, as use of sunscreen alone, despite a high factor, is likely to leave them unprotected. Interestingly, in this study the quantity of products applied was monitored and did meet the recommended $2 \mathrm{mg} / \mathrm{cm}^{2}$. Thus, despite an appropriate quantity, effective protection was not achieved. In addition to protective clothing and shade seeking, a higher SPF index should be formulated for fair-skinned individuals exposed to extreme UV radiation.

Regarding the methodology of the study, other authors have assessed the reliability of colorimetry and visual assessment: Wright et al. [9] assessed visual assessment of skin color by clinicians and instrumental colorimetry, finding a strong correlation, while Basketter et al. reported that visual erythema assessment was sensitive and reproducible [10]. We found a strong correlation between clinically assessed erythema and instrumentally measured erythema $\left(a^{*}\right)$, as demonstrated by Spearman's rho (0.663) - a value of 1.0 would represent a perfect positive correlation and a value of zero would indicate no association. This lends support to the methods used, suggesting that they are meaningful and consistent with real solar-induced skin damage. To provide further data, an addition to future studies could be to integrate assessment of early and late induced pigmentation to discriminate small differences among several sunscreens.

The location of the study, in Tamarin, Mauritius, at $20^{\circ} 9^{\prime}$ south of the equator in the southern Indian ocean, was chosen to test the product under more intense solar radiation. Of course, the efficacy results observed should not be used to encourage sun exposure in such conditions, and sun safety recommendations in their respective national and international versions should still be followed [11, 12], taking into account habits and behaviors that may affect exposure intensity [13]. Of note, we can infer from the results that individuals with phototype II (or lower) are unlikely to be sufficiently protected by SPF50 and SPF50+ products in such extreme conditions at the recommended 2-hourly application rate, even when application technique is optimal. Instead, we must consider what specific recommendations should be given to this population on their UV response.

The design of this study, being intra-individual, ensured that variability was reduced. We further wanted to address some of the weaknesses inherent to standard SPF testing, by performing an outdoor study that would expose subjects to the full solar spectrum, thus making the results more representative of real-life solar exposure. Few studies using outdoor methods have been published [14-17]. The approach used in this study bears resemblance to established methods used for other dermatological assessments: variations of Dumas and Scholtz's plaque test [18-20] are frequently used to assess the efficacy of multiple topical treatments while minimizing variability by comparing intra-individual outcomes. Here we wanted to utilize the benefits of this approach, and we judge that the results add robustness to the product's efficacy claims. The limitations of the study relate to those aspects of real-life outdoor conditions that are highly difficult to control for, including weather conditions, accurate recording of solar exposure, and activities performed by users.

Although the product was designed to be used on the face, we tested it on the back to allow space for all the test areas. Testing of 
similar-sized areas on the face would have posed some practical problems, since intra-individual variation in skin type (oily, dry, or sensitive areas in different facial zones) may have affected responses to both sun exposure and product tolerability. We would like to mention, though, that the product has previously been tested in an outdoor study with a split-face design [7].

Correct use of sunscreens and protective measures in real life requires ongoing public education, but key to ensuring adequate use of sunscreens is the creation of products that are pleasant to use. This product, designed with this in mind, has been demonstrated in this study to sustain a very high level of solar protection in conditions more representative of real-life exposure.

This study did not assess the effects of user education or cosmetic formulation on sunscreen application in real life, although clearly these are important areas in which further investigation may be warranted.

\section{CONCLUSION}

The methodology presented represents an interesting design with good correlation of clinical and instrumental assessments. It provides a closer approximation of real-life solar exposure, circumventing some of the limitations inherent to standardized SPF testing and allowing a relative ranking of multiple products simultaneously, which may help guide consumers' and dermatologists' choice of sunscreen. In outdoor conditions of very high to extreme solar radiation, the investigational water-based broad-spectrum SPF50+ was significantly superior to the reference standard SPF15 and similar to the SPF50+ control products in terms of photoprotection.

\section{ACKNOWLEDGEMENTS}

We thank the participants of the study.

Funding. The sponsor (Isdin) funded the costs of performing the study and all publication costs.
Medical Writing Assistance: Jane Marshall, a freelance medical writer, assisted in drafting the manuscript, with costs funded by the sponsor.

Authorship. All named authors meet the International Committee of Medical Journal Editors (ICMJE) criteria for authorship for this article, take responsibility for the integrity of the work as a whole, and have given their approval for this version to be published. All authors had full access to all of the data in this study and take complete responsibility for the integrity of the data and accuracy of the data analysis.

Disclosures. Corinne Granger is an employee of Isdin. Javier Bustos is an employee of Isdin. Carles Trullàs is an employee of Isdin. Yolanda Sola is an independent consultant for Isdin. Philippe Andres is an independent consultant for Isdin. Jean Krutmann is an independent consultant for Isdin. Thierry Passeron is an independent consultant for Isdin. Muzzammil Hosenally is an employee of Centre International de Développement Pharmaceutique which received funding from Isdin to run the statistical analysis of this study.

Compliance with Ethics Guidelines. The study was approved by an independent ethics committee (Independent Ethics Committee, King George V Corner, Floreal). The study was conducted in line with the principles of the Declaration of Helsinki and its subsequent updates, the International Conference on Harmonization Good Clinical Practices, and local laws. All subjects provided written informed consent to participate.

Data Availability. The datasets analyzed during the current study are available from the corresponding author on reasonable request.

Open Access. This article is distributed under the terms of the Creative Commons Attribution-NonCommercial 4.0 International License (http://creativecommons.org/licenses/ by-nc/4.0/), which permits any noncommercial use, distribution, and reproduction in any 
medium, provided you give appropriate credit to the original author(s) and the source, provide a link to the Creative Commons license, and indicate if changes were made.

\section{REFERENCES}

1. Lautenschlager S, Wulf HC, Pittelkow MR. Photoprotection. Lancet. 2007;370(9586):528-37.

2. Sklar LR, Almutawa F, Lim HW, Hamzavi I. Effects of ultraviolet radiation, visible light, and infrared radiation on erythema and pigmentation: a review. Photochem Photobiol Sci. 2013;12(1):54-64.

3. Azurdia RM, Pagliaro JA, Diffey BL, Rhodes LE. Sunscreen application by photosensitive patients is inadequate for protection. $\mathrm{Br} \mathrm{J}$ Dermatol. $1999 ; 140(2): 255-8$.

4. International Organization for Standardization. ISO 24444:2010 Cosmetics-Sun protection test methods-In vivo determination of the sun protection factor (SPF). https://www.iso.org/standard/46523. html. Accessed 19 June 2019.

5. US Food and Drug Administration. Labeling and effectiveness testing; sunscreen drug products for over-the-counter human use. Final rule, 21CRF Parts 201 and 310. Federal Register, vol. 76, no. 117, pp. 35620-35665, 2011. https://www.govinfo.gov/ content/pkg/FR-2011-06-17/pdf/2011-14766.pdf. Accessed 19 June 2019.

6. International Organization for Standardization. ISO 24442:2011 Cosmetics-Sun protection test methods-In vivo determination of sunscreen UVA protection. https://www.iso.org/standard/46521.html. Accessed 19 June 2019.

7. Granger C, Sola Y, Gilaberte Y, Trullas C. Outdoor testing of the photoprotection provided by a new water-based broad-spectrum SPF50+ sunscreen product: two double-blind, split-face, randomised controlled studies in healthy adults. Clin Cosmet Investig Dermatol. 2019;12:461-7.

8. Lowe NJ, Dromgoole SH, Sefton J, Bourget T, Weingarten D. Indoor and outdoor efficacy testing of a broad-spectrum sunscreen against ultraviolet A radiation in psoralen-sensitized subjects. J Am Acad Dermatol. 1987;17(2 Pt 1):224-30.

9. Wright CY, Wilkes M, du Plessis JL, Reeder AI, Albers PN. In multiple situational light settings, visual observation for skin colour assessment is comparable with colorimeter measurement. Skin Res Technol. 2016;22(3):305-10.
10. Basketter D, Reynolds F, Rowson M, Talbot C, Whittle E. Visual assessment of human skin irritation: a sensitive and reproducible tool. Contact Dermatitis. 1997;37(5):218-20.

11. British Association of Dermatologists. Sunscreen and sun safety factsheet 2013. http://www.bad.org. $\mathrm{uk} /$ for-the-public/sun-awareness-campaign/down loadable-leaflets-and-posters. Accessed 19 June 2019.

12. World Health Organization. WHO INTERSUN-the global UV project. 2006. Sunshine and health: how to enjoy the sun safely. https://www.who.int/uv/ publications/sunshine-and-health/en/. Accessed 19 June 2019.

13. Diffey B. Time and place as modifiers of personal UV exposure. Int J Environ Res Public Health. 2018;15(6):1112.

14. Williams JD, Maitra P, Atillasoy E, Wu MM, Farberg AS, Rigel DS. SPF $100+$ sunscreen is more protective against sunburn than SPF 50+ in actual use: results of a randomized, double-blind, split-face, natural sunlight exposure clinical trial. Am Acad Dermatol. 2018;78(5):902-910.e2.

15. Ou-Yang H, Jiang LI, Meyer K, Wang SQ, Farberg AS, Rigel DS. Sun protection by beach umbrella vs sunscreen with a high sun protection factor: a randomized clinical trial. JAMA Dermatol. 2017;153(3):304-8.

16. Russak JE, Chen T, Appa Y, Rigel DS. A comparison of sunburn protection of high-sun protection factor (SPF) sunscreens: SPF 85 sunscreen is significantly more protective than SPF 50. J Am Acad Dermatol. 2010;62(2):348-9.

17. Lott D. Testing SPF 15-100, indoor vs. outdoor. Cosmet Toiletries Mag. 2013;128(9):638-647.

18. Dumas KJ, Scholtz JR. The psoriasis bio-assay for topical corticosteroid activity. Acta Derm Venereol. 1972;52(1):43-8.

19. Queille-Roussel C, Hoffmann V, Ganslandt C, Hansen KK. Comparison of the antipsoriatic effect and tolerability of calcipotriol-containing products in the treatment of psoriasis vulgaris using a modified psoriasis plaque test. Clin Drug Investig. 2012;32(9):613-9.

20. Lee CS, Koo J. The efficacy of three class I topical synthetic corticosteroids, fluocinonide $0.1 \%$ cream, clobetasol $0.05 \%$ cream and halobetasol $0.05 \%$ cream: a Scholtz-Dumas bioassay comparison. J Drugs Dermatol. 2009;8(8):751-5. 